\title{
Structural Operational Integrity - The Comprehensive Quality Characteristic of Structure
}

\section{Yuting He ( $\nabla$ heyut666@126.com )}

Air Force Engineering University

\section{Research Article}

Keywords: structure, structural integrity, structural operational integrity, structural comprehensive quality characteristic, characterization, structural optimum design

Posted Date: March 7th, 2022

DOI: https://doi.org/10.21203/rs.3.rs-1322499/v1

License: (c) (i) This work is licensed under a Creative Commons Attribution 4.0 International License. Read Full License 


\title{
Structural Operational Integrity - The Comprehensive Quality
}

\section{Characteristic of Structure}

\author{
Yuting He
}

Air Force Engineering University; Xi'an 710038, China;

Email: hytmems@126.com

\begin{abstract}
:
Carbon reduction has been closely bound up with economy and social development. So, a higher demand for structural integrity is brought to meet the different requirements of equipment, ma-chines and infrastructures. For structures, one of their general quality characteristics such as reliability, safety, maintainability and so on, only reflects a certain aspect of quality characteristics. In this paper, After discussing equipment integrity which can be classified into equipment manufacture integrity, equipment storage integrity and equipment operational integrity along the life cycle time, from the perspective of performing and completing the functions and missions of structures, the putting forward process of structural operational integrity(SOI) is discussed, and the basic concept of structural operational integrity is shown: it is the attribute which exists when a structure is sound and unimpaired in full service or whole operational processes, with the statement that structural operational integrity can be used to describe the comprehensive quality characteristics of structures in their whole service or operational processes presented. Then, structural operational integrity is categorized into structural static operational integrity(SSOI) and structural dynamic operational integrity(SDOI), While definitions of them are given as follows: structural static operational integrity is the status which exists when a structure is sound and unimpaired in service or operational process, and structural dynamic operational integrity is the ability which exists when a structure is sound and unimpaired while providing the desired levels of structural durability, supportability, safety, performance, survivability and recoverability during service period or in operational processes. Furthermore, the characterizations of structural static operational integrity(SSOI) and structural dynamic operational integrity(SDOI) are presented, while the measurement methods of durability, supportability, safety, performance, survivability and recoverability of structures are discussed. Finally, it states that PHM technologies can improve structural operational integrity, and the optimum design method of structures based on structural operational integrity is described with an aircraft wing structure design as an example. With the employment of structural operational integrity, the structural design idea will be developed. It can help us get lighter, low life cycle cost, durable, fast recovery and more reliable structures. And structural operational integrity can be applied to other engineering structures such as building structures, bridge structures, infrastructure bodies, etc.
\end{abstract}

\section{Keywords:}

structure; structural integrity; structural operational integrity; structural comprehensive quality characteristic; characterization; structural optimum design 


\section{Introduction}

Usually, as same as equipment, the general quality characteristics of equipment structure in service include reliability, maintainability, supportability, safety, and testability, etc. ${ }^{[1]}$. However, they all reflect the characteristics of equipment structure from one perspective, and they can't tell the overall quality characteristic of the structure while the relationship between them is not well explained. Often the user's quality requirements for structure are focused on three points: meeting the performance requirements, being performance-stable and being service-durable during service period or in operational processes. In reference [2], from the perspective of performing and completing the functions and missions for the military aircraft structures, as the development of traditional aircraft structural integrity (ASI), the author presented the concept of battle integrity for military aircraft structures. In fact, the battle integrity for military aircraft structures means the operational integrity for aircraft structures in the military operational processes as well as that for civil aircraft structures in service. So, the concept of operational integrity can be further applied to all aircraft structures and other equipment structures during their service periods, including civil or military aircraft structures and other equipment structures ${ }^{[3]}$. Therefore, structural operational integrity concerns the overall quality of structure and can be used to characterize the comprehensive quality characteristic of structure. The comprehensive quality characteristics of structures are often determined during design period, solidified during manufacture period, maintained during service periods. They are the inherent attributes of the equipment structures which are manifested in the entire life cycles. Obviously structural operational integrity concerns the structural strength, stiffness, durability, damage tolerance, force protection, fast repair and so on, which affect the safe use, cost and scale of structures in service or operational processes.

So, in this paper, the putting forward process and concept of structural operation-al integrity (SOI) are expounded firstly. As is stated that the SOI reflects the comprehensive quality level of structure, the relationship between structural operational integrity and traditional general quality characteristics of structures is discussed. Also, it is discussed how to categorize the structural operational integrity. Furthermore, the characterization of structural operational integrity is presented; the measurement methods of durability, supportability, safety, performance, survivability and recoverability of structures are illustrated. Finally, the optimum design method of structural operational integrity is described; the effect of PHM technologies on structural operational integrity is also discussed. With the employment of structural operational integrity, the structural design idea will be improved and lighter, easy-repair, low life cycle cost and more reliable structures could be designed. And this will be very good for carbon reduction in transportation, production, construction, etc.

\section{The putting forward process and concept of structural operational integrity}

Essentially, Integrity means an undivided or unbroken completeness or totality with nothing wanting. Structural integrity goes back as far as recorded history. Structural integrity is not just a case of good design, it needs to be maintained for the life of a structure. This requires inspection and maintenance at periodic intervals. The concept of structural integrity was first proposed by the United States Air Force in $1954{ }^{[3]}$, and gradually developed and improved with a series of accidents in the United States Air Force. The 
corresponding standards as well as Aircraft Structural Integrity Program (ASIP) has more than ten supplements and revisions, such as ASIP in the United States, MIL-STD-1530D ${ }^{[4]}$ released in 2016, ASIP in China, GJB775.A - $2012^{[5]}$, etc. Aircraft structural integrity is the first concept of integrity and it plays a very important role in aircraft integrity. Aircraft structural integrity is the condition which exists when a structure is sound and unimpaired while providing the desired level of structural safety, performance, durability, and supportability ${ }^{[4]}$. In 2021, The Welding Institute stated that structural integrity is an engineering field that helps ensure that either a structure or structural component is fit for purpose under normal operational conditions and is safe even should conditions exceed that of the original design ${ }^{[6]}$.

It can be seen that the traditional aircraft structural integrity is mainly oriented to training stage of military aircraft, which is the most basic requirement of military air-craft structures in service, but it is not suitable for battling stage of military aircraft structures. For civil aircraft structures, the traditional aircraft structural integrity is suitable for normal service but is not suitable for accidental events. The traditional structural integrity mainly focuses on load/environment spectrum measurements during the implementation of force management. The purpose is to update the conclusions of full-scale test, so as to realize the accurate life determination of structures. However, in battling stage, the load/environment spectrum of aircraft structure is un-predictable, and there is no time to update the conclusions of aircraft structure life, so the impact of structural battle damages should be taken into account. Therefore, it is urgent to develop the traditional structural integrity to suit the battle environments for military aircraft structures and accidental conditions for civil aircraft structures.

In wartime, the main task of structural integrity of military equipment should be to maintain the equipment structure's sustained combat capability under specified battle conditions. Therefore, in order to meet the requirements in wartime, we should consider the indicators that affect the sustained combat capability while reflecting the inherent characteristics of structures. For this reason, the structural operational integrity should be developed. In fact, from the view of life cycle time, the equipment operational functions should be assigned in design processes, realized in manufacturing processes, and maintained in storage processes and operational processes, just shown in Figure 1.

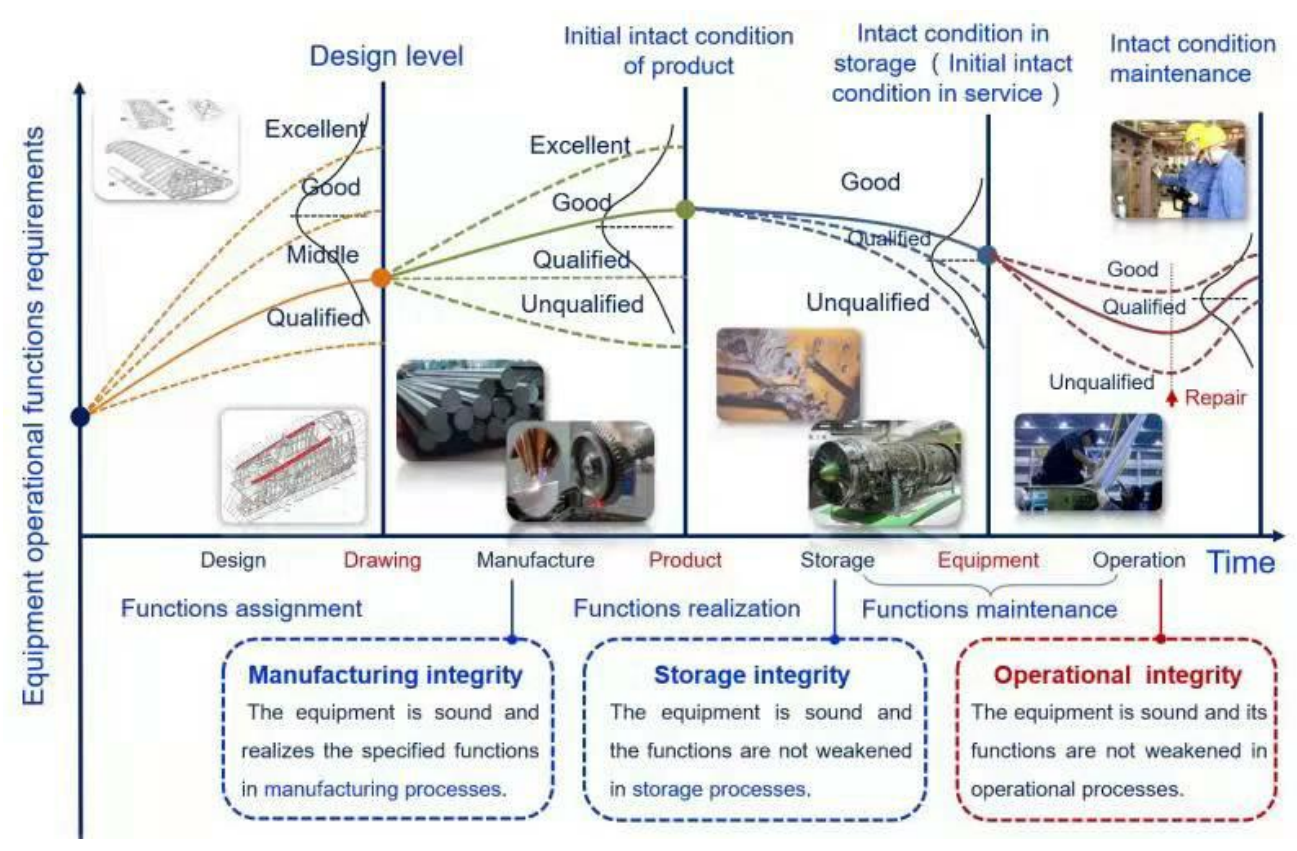


Figure 1. Equipment integrity classification along the life cycle time.

At the end of design processes, we can get the design drawings. After the manufacturing, we can get the products. After the storage processes (long or short time), when the customers get the products, they are usually called equipment. When the equipment is operated in operational processes, it can be apolitical in order to function and perform effectively. Usually, the design drawings will reflect the design level of designers. After the product manufactured, it will show the level of initial intact condition of product itself. After the storage period, the product will tell the level of intact condition in storage ( or the level of initial intact condition of equipment in service ). And, in the operational processes, the level of intact condition of equipment should be maintained through some necessary maintenance work. In order to keep the operational functions of equipment in its operational processes, equipment integrity should be maintained. Along the life cycle time, equipment integrity can be categorized into manufacturing integrity which refers to the attribute that the equipment is sound and realizes the specified functions in manufacturing processes, storage integrity which refers to the attribute that the equipment is sound and the functions are not weakened in storage processes and operational integrity which refers to the attribute that the equipment is sound and its functions are not weakened in operational processes. The equipment operational integrity, equipment operational suitability, and equipment operational effectiveness are three basic attributes of any equipment ${ }^{[7,8]}$. Equipment operational suitability refers to the degree of satisfaction of the equipment on the battlefield. It is related to reliability, maintainability, supportability, safety, compatibility, and interoperability factors, including transportability, environmental adaptability, documentation, personnel, and training. The equipment operational effectiveness is the overall extent to which the representative personnel complete a mission with the equipment in a planned or expected equipment operation environment (such as natural environment, electromagnetic environment, battlefield threat, etc.). And it can be seen that the equipment operational integrity is the basis for the equipment operational suitability and equipment effectiveness.

Obviously, as the main part of equipment, structure should maintain its integrity, structural integrity, in order to maintain equipment integrity. Similarly, along the life cycle time of structure, structural integrity can also be categorized into structural manufacturing integrity, structural storage integrity and structural operational integrity. If we want to operate the structures successfully in service or operational processes, something should be focused on structural operational integrity.

For aircraft, the structural operational integrity is proposed in reference [2] and [3], and defined as the attribute which exists when a structure is sound and unimpaired while providing the desired levels of structural durability, supportability, safety, performance, survivability and recoverability in service. It means that under the required level of structural durability, supportability, safety, performance, survivability and recoverability, aircraft structure can be operated normally and its function is not weakened during service period, including the normal training conditions and the battling or the accidental conditions for military or civil aircraft. Compared with the concept of traditional aircraft structural integrity, structural operational integrity adds the requirements of survivability and recoverability for aircraft structures, which is shown in Figure 2. 


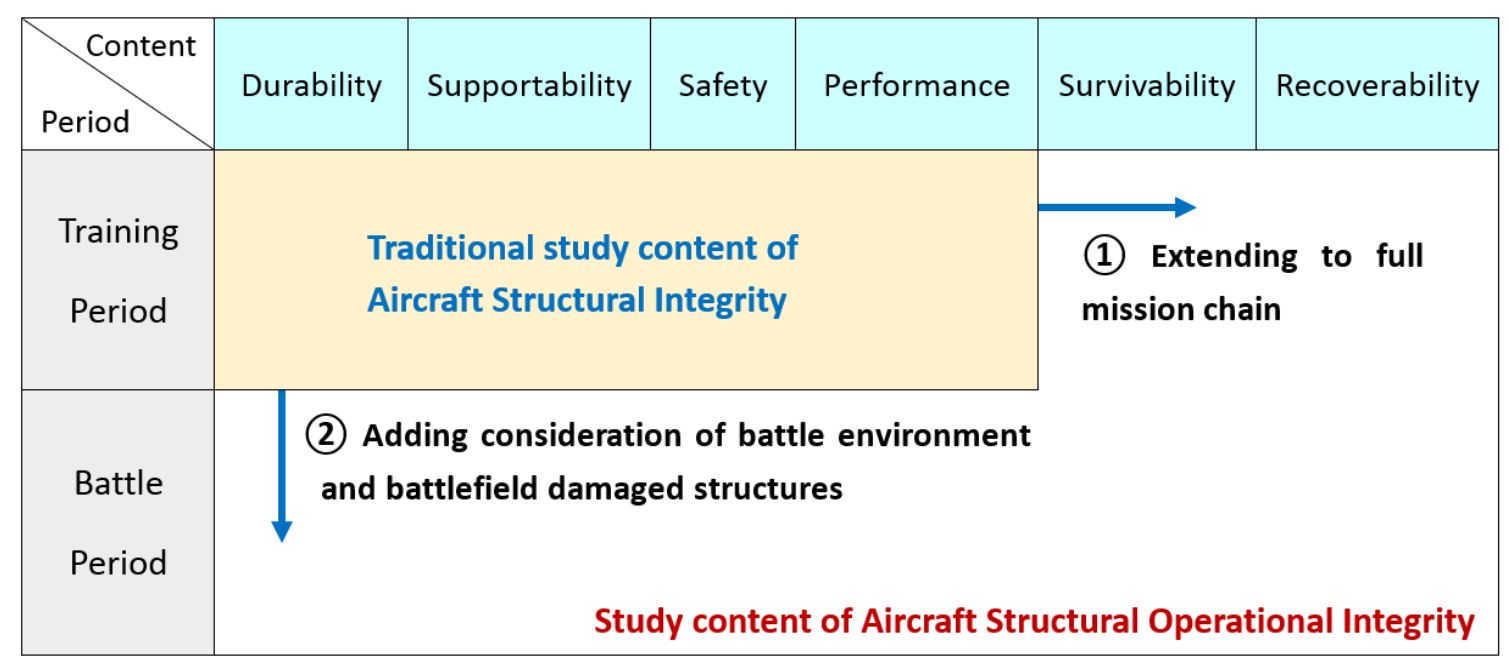

Figure 2. Content of SOI is the development of the content of traditional ASI.

Actually, from the perspective of consideration of full-service period of aircraft, including the training period and battling period for military aircraft as well as the normal conditions and accidental conditions for civil aircraft, structural operational integrity refers to the basic attribute which exists when a structure is sound and unimpaired in full service or whole operational processes. As a typical concept, for any kind of equipment, structural operational integrity (SOI) is the attribute which exists when a structure is sound and unimpaired in full service or whole operational processes.

\section{Categorization and characteristics of structural operational integrity}

Generally speaking, structural operational integrity, as an attribute of structure, includes static attribute and dynamic attribute, showing the status and ability of structure separately. So, structural operational integrity can be categorized into structural static operational integrity (SSOI) and structural dynamic operational integrity (SDOI). Structural static operational integrity can be defined as the status which exists when a structure is sound and unimpaired in service or operational process, and structural dynamic operational integrity can be defined as the ability which exists when a structure is sound and unimpaired while providing the desired levels of structural durability, supportability, safety, performance, survivability and recoverability during service period or in operational processes. It can be seen that structural static operational integrity concerns the status of structure at the exact moment while structural dynamic operational integrity concerns the ability of structure to keep sound and unimpaired during one exact period. It is clear that the issues about structural operational integrity discussed above and in [2,3] refer to structural dynamic operational integrity (SDOI).

Also, the basic characteristics of structural operational integrity can be summarized as objectivity, relativity, randomness and controllability. Objectivity means that SOI is an objective structural attribute, which can be measured by some means. Relativity means that SOI matches the exact task and operating environment it undertakes, and it makes no sense when leaving the corresponding task and operating environment. Randomness means that SOI also has random characteristics due to the quality of the structure itself, the randomness of task and operating environment. The methods of probability and statistics can often be used to describe SOI. Controllability means that SOI can be controlled by certain measures, which will be 
discussed in the next work.

\section{Structural operational integrity represents the comprehensive quality characteristic}

\section{of structure}

4.1. Structural operational integrity shows the comprehensive quality characteristic of structure

For structure, it has some special performances, such as strength, rigidity, stability, sealability and movement. But in service, failures always affect the functions and performances of structure, as well as the general quality characteristics of it, such as reliability, safety, maintainability, testability, supportability and so on. That is to say, failures always affect the operational integrity and comprehensive quality characteristic of structure in service. Taking the aircraft structures as an example, the different failure modes and their effects on structural performances and general quality characteristics as well as some remarkable events are shown in Figure $3^{[5,9-11]}$.

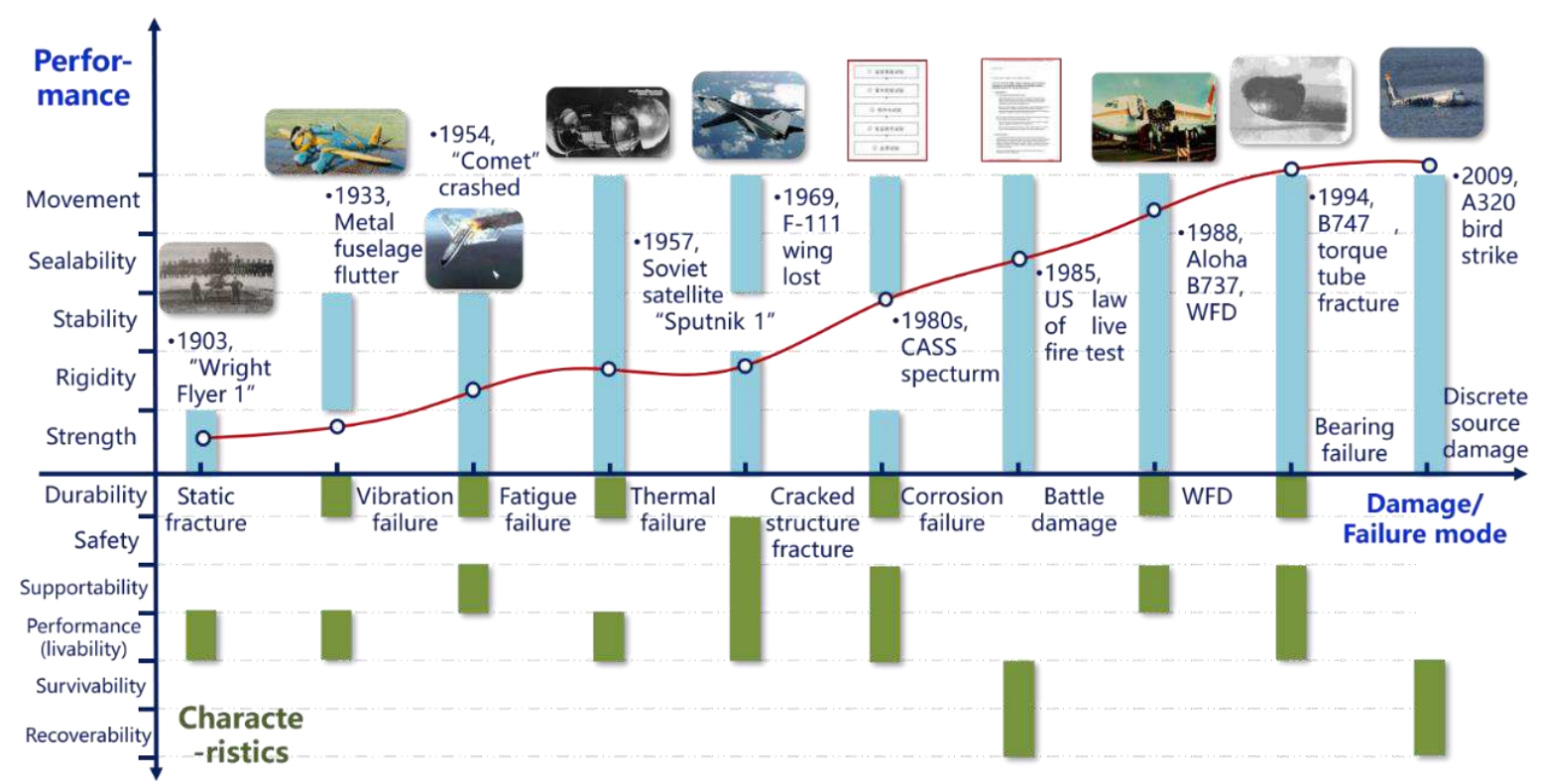

Figure 3. The effects of failure modes on aircraft structural performances and characteristics.

It is known that general quality characteristics of the aircraft structures refer to reliability, safety, maintainability, testability, supportability and so on. Also, survivability and recoverability are general quality characteristics of aircraft structures. They are gradually developed and improved around the problem of aircraft structure failures during service period ${ }^{[14]}$, which are reflected from different aspects. The main purpose of these quality characteristics of the aircraft structure is to ensure that the aircraft structure can meet the usual reserve and use requirements during the service period, to ensure stable working statuses and technical performances, and to reduce life cycle costs ${ }^{[15-16]}$. Indeed, the environmental adaptability of aircraft structure belongs to aircraft structural operational suitability, which is not discussed here. In general, the six indexes of aircraft structural operational integrity are mostly the same as those general quality characteristics of aircraft structure. However, comparing both of them, the general quality characteristics of 
aircraft structure reflect a series of measurements of the general quality level of aircraft structure, each of which indicates the quality characteristic from a certain aspect of aircraft structure. Strictly speaking, environmental adaptability is part of aircraft structural operational suitability, and its requirements are higher than quality requirements. The application characteristics of aircraft structural operational integrity are more distinctive. It adds survivability and recoverability indexes of aircraft structure as new parameters to aircraft structural operational integrity, taking aircraft structural ability to complete operational missions as a traction, which reflects the comprehensive quality characteristic of aircraft structure when aircraft can be successfully operated to complete the required missions in service. Aircraft structural operational integrity is a measurement of the available state or ability of an aircraft structure, where durability concerns reliability and economical effectiveness. Therefore, it can be seen that aircraft structural operational integrity comprehensively reflects the quality characteristic requirements, such as the satisfied performances of aircraft structure, the stable performances of aircraft structure and the durable service of aircraft structure. Obviously, structural operational integrity is not only related to general quality characteristics of structure, but also has its own distinct characteristic, which represents the comprehensive quality characteristic of a structure.

It should be noted that, as one of the general quality parameters of structure, survivability refers to the structural ability to avoid and withstand hostile or accidental harmful environments without permanently degrading its performances to perform specified missions. Military aircraft susceptibility and vulnerability are the two main factors that determine the survivability of aircraft ${ }^{[17,18]}$. The survivability of a civil equipment structure can be used to characterize its capability to avoid and withstand accidental threats such as bird strikes, hail or lightning strikes etc. without permanently degrading its performances to perform specified transportation tasks.

About another new general quality parameter, recoverability refers to the ability of structure to recover to the required functional state to complete the specified tasks after repairing the unconventional damages (including accidental damages, battle damages, etc.) according to the specified procedures and methods under the specified conditions within the specified time ${ }^{[19]}$. Structural recoverability concerns the repair of unconventional damages such as accidental damages and battle damages. In wartime, the recoverability in the field level is equivalent to battle damage reparability ${ }^{[20-21]}$. The degree of structural recoverability is reflected from two aspects. One is whether the structure can be easily recovered in the event of damages, especially unconventional damages, which is reflected on the supporting equipment, recovery technologies, manpower, and time required for recovery; another is whether the structure can be repaired economically in case of the same damages, which is reflected on the spare parts, consumables and costs needed in recovery processes. When the cost and time required for the repair exceeds a certain limit, the structure recovery is not cost-effective, and the structure is considered unrecoverable at this time.

Something should be pointed out here. Recoverability and maintainability of structure are inherent characteristics of a structure which are determined through the design and manufacture processes, and they have many common requirements: such as accessibility, interchangeability, and error proofing design, etc. However, there are obvious differences between recoverability and maintainability. Maintainability concerns 
the natural failures of the structure during its normal service period, including failures due to reliability of it, and human errors. The focus of maintainability is on "maintain and repair", and generally there are expected standard maintenance or troubleshooting schemes; while recoverability concerns unexpected damage of a structure, including accidental damages (such as bird strikes, lightning strikes, ground collisions, etc.) and battle damages (such as weapon damages and secondary damages, etc.), with focus on "repair and recover". It emphasizes on repair measures for unforeseen damages. In addition, compared with maintainability design, recoverability design should pay more attention to the employment of modular design ideas, which is more conducive to the ability to easily and quickly recover damaged parts of structures.

\subsection{The influence of various general quality parameters on structural operational integrity}

In order to analyze the influence of various general quality parameters on structural operational integrity, the equipment mission chain in whole operational processes should be mentioned. Here aircraft is employed as an example for analyses. When the military aircraft is placed in the battlefield, its combat mission chain can be simply summarized as follows: aircraft assembly $\rightarrow$ aircraft sortie generate $\rightarrow$ aircraft cruise $\rightarrow$ aircraft entry (to the battlefield) $\rightarrow$ aircraft combat $\rightarrow$ aircraft withdrawal (from the battlefield) $\rightarrow$ aircraft return. Then, the aircraft reassembly, and enters the next combat cycle until the battle is finished. The civil aircraft's operational processes are much simpler as follows: aircraft assembly $\rightarrow$ aircraft departure $\rightarrow$ aircraft cruise $\rightarrow$ aircraft return. And then, the next usage cycle continues. So, military aircraft will be mainly discussed afterwards. In the mission chain of military aircraft structure, traditional aircraft structural integrity indicators (durability, supportability, safety and performance) reflect whether the structure can be operated well before combat (i.e., the normal training) for military aircraft; while the aircraft structural operational integrity indicators (survivability and recoverability added) reflect whether the aircraft structure can continue to perform and complete combat tasks in wartime. The influence of military aircraft structural operational integrity on mission chain is in 6 aspects as shown in Figure 4.

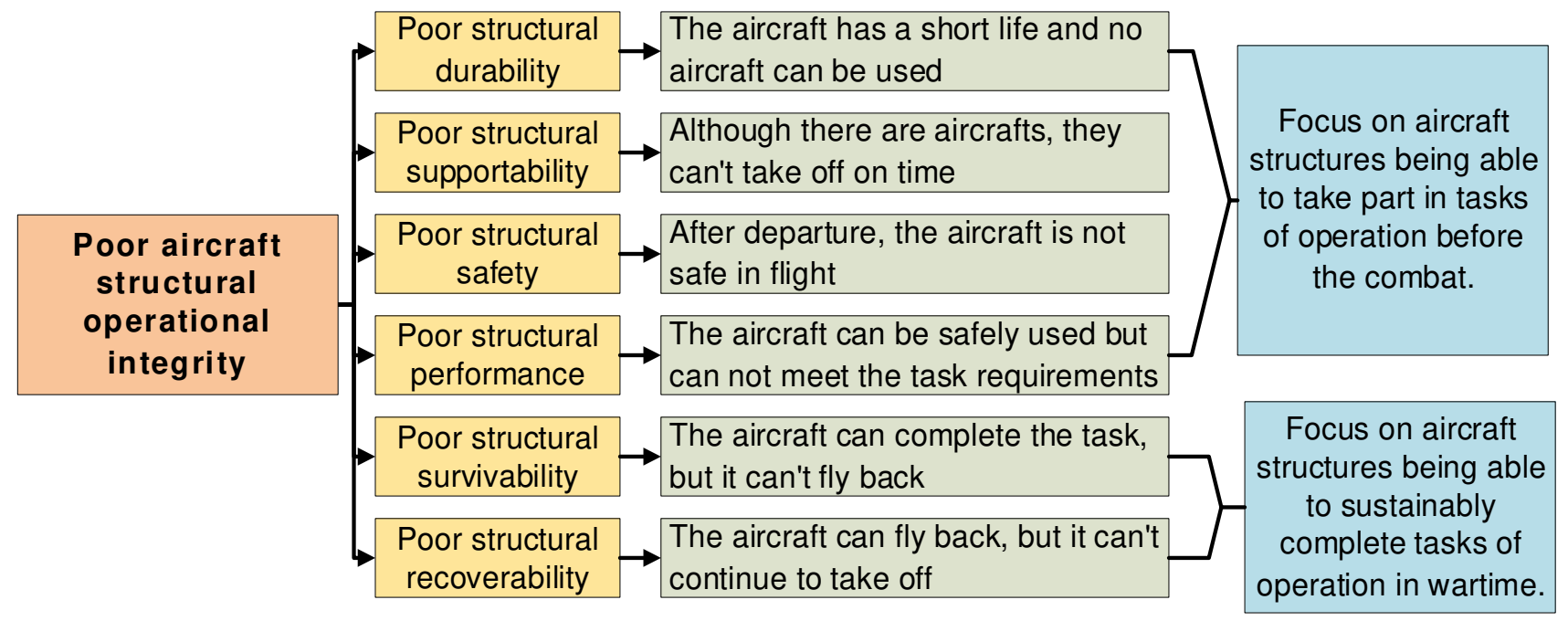

Figure 4. The influence of military aircraft structural operational integrity on operational chain.

Of course, the actual situation is much more complicated and serious than the descriptions in last figure, because some index elements of aircraft structural operational integrity work continuously in several stages 
of aircraft structural operating processes.

From the discussion above, it can be seen clearly that, for structural operational integrity, the deficiency of index factors in any aspect will lead to the overall low level of it. That is to say, the structural operational integrity index system conforms to "cask effect", which must be considered comprehensively and coordinately in the whole life cycle of structures. From the discussion it can be seen that, if one wants to get high structural operational integrity, structural operational integrity index system must be optimally matched. It is to say that structure must be optimally designed to get high structural operational integrity. Before this, characterization of structural operational integrity should be understood.

\section{Characterization of structural operational integrity}

Generally speaking, structural operational integrity (SOI) can be characterized by means of fundamental readiness rate of structures in the fleet with the same type, Rs, structural fundamental health degree of a structure, HS(t), and structural operational integrity degree, Iso. Three of them will be discussed as follows. It should be noticed that a kind of structures or the same type of structures are analyzed when discussing the characterization of structural operational integrity and other characteristics of structures in the rest of this paper.

\subsection{Characterization of structural static operational integrity(SSOI)}

From the discussion above, structural static operational integrity concerns the status of structure at the exact moment. So, structural static operational integrity can be directly reflected by the fundamental readiness rate of structures, $R_{s}$, which represents the ratio of the number of structures in sound state to the total number of structures in the fleet with the same type under a specified operational environment, supporting environment and management environment.

$$
R_{S}=\frac{E_{S-\text { intact }}}{E_{\text {s-total }}}
$$

where $R_{S}$ is structural fundamental readiness rate; $E_{S \text {-intact }}$ is the number of structures in sound state; $E_{S-t o t a l}$ is the total number of structures in the fleet with the same type. In fact, structural fundamental readiness rate can be categorized into structural fundamental readiness rate $R_{s t}$ in peacetime or normal service environments and structural fundamental readiness rate $R_{s b}$ in wartime or accidental service environments. Their values are generally different. It can be seen that the values of structural fundamental readiness rate will usually be changed along the service time of structures.

On the other hand, PHM technologies are widely studied and some of them have been applied in structural health monitoring (SHM), while structural health degree was used to express the health status of a structure ${ }^{[22]}$. Structural fundamental health degree is the level at which the structure can remain sound (or work normally) and its functions are not weakened when the structure performs the specified tasks under the specified operational environment, supporting environment and management environment, which can be represented by formula (2).

$$
H_{s}(t)=1-L_{a}(t) / L_{c}(t)
$$

where $t$ is the point in time; $H_{S}(t)$ is structural health degree, which is a function of the point in time; $L_{a}(t)$ is the real crack length of structure when the structure is in work. $L_{c}(t)$ is the critical crack length of 
structure when the structure is in failure with fracture. Structural fundamental health degree covers from 0 to 1, which reflects the healthy level of a structure at a moment. Finally, structural health status can be determined according to the value of structural fundamental health degree and classified into healthy status, sub-healthy status and unhealthy status. For example, for a structural member, it can be determined as in healthy status when $H_{s}(t)$ is equal to or greater than 0.9 , in sub-healthy status when $H_{s}(t)$ is equal to or greater than 0.3 and less than 0.9 , and in unhealthy status when $H_{s}(t)$ is less than 0.3 , as shown in Figure 5.

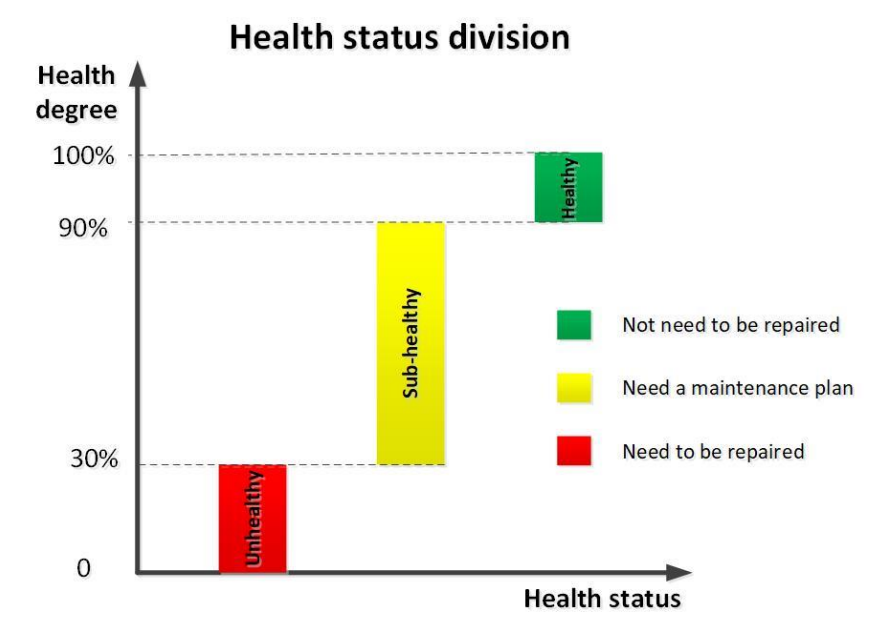

Figure 5. Healthy status division of a structural member.

Usually, it is unnecessary to repair the structure when it is in healthy status; it is necessary to make a plan to repair the structure when it is in sub-healthy status; it is equally necessary to repair the structure at once when it is in unhealthy status.

Basically, the status of a structure being sound and unimpaired means the state of a structure in health. So, the structural static operational integrity can also be characterized by means of structural fundamental health degree, $H_{S}(t)$. When a structure is in healthy status and sub-healthy status, it can work normally, and the structure can be thought as in sound state. When structure is in unhealthy status, it can't work normally and must be repaired at once, and the structure can be thought as in soundless state. Furthermore, the fundamental readiness rate of structures, $R_{S}$, can be calculated based on the number of structures in sound state and the total number of structures in the fleet with the same type. Also, if necessary, only the structures in health can be thought as in sound state, while the structures in sub-healthy status and unhealthy status can be thought as in soundless state. Then the fundamental readiness rate of the structures, $R_{s}$, can be calculated consequently. It should be pointed out that PHM technologies can improve the fundamental readiness rate of structures, $R_{S}$, because it can improve a lot, i.e., the maintaining efficiency. That is to say that PHM technologies can improve structural operational integrity.

\subsection{Characterization of structural dynamic operational integrity (SDOI)}

Structural dynamic operational integrity concerns the ability of a structure to keep sound and unimpaired during one exact period. In order to effectively represent structural dynamic operational integrity (SDOI), structural operational integrity degree, Iso, can be given, and then structural dynamic operational integrity can be measured ${ }^{[3]}$. Structural operational integrity degree is the probability that the structure can remain sound (or work normally) and functions are not weakened when the structure performs the specified 
tasks within the specified time under the specified conditions, which can be represented by formula (3). According to the definition of structural dynamic operational integrity, the structural ability keeping sound and unimpaired is determined by structural durability, supportability, safety, performance, survivability and recoverability. Therefore, structural operational integrity degree, Iso, is comprehensively characterized and functioned by structural durability degree Us, availability degree As, safety degree Ss, livability degree Cs, survivability degree $S_{s u}$ and recoverability degree $R_{s c}$, which can be expressed as follows:

$$
I_{s o}=P\left\{\tau>t_{0}\right\}=f\left(U_{s}, A_{s}, S_{s}, C_{s}, S_{s u}, R_{s c}\right)
$$

where, $t_{0}$ is the specified time; $\tau$ indicates the time during which the structure can be sound and unimpaired; $U_{s}$ is structural durability degree; $D_{s}=1-U_{s}$, which is structural damage degree, a quantitative measure of the durability damage of structure when it reaches the specified time $t ; A_{s}$ is availability degree, which is used to measure structural supportability; $S_{S}$ is structural safety degree, which is used to measure structural safety and represents the probability without accidents when the structure completes the specified task under the specified conditions throughout the specified time cycle $C_{s}$; is structural livability degree; $F_{s}=$ $1-C_{s}$, the failure rate of aircraft structures, which is the probability of structural failure when the ability of structure to bear the loads is equal to or less than the loads carried by the structure; $S_{s u}$ is structural survivability degree, which refers to the probability of structure being able to keep working with the damage due to various weapons, unexpected accidents and non-calculated loads; $R_{s c}$ is structural recoverability degree, which means the probability where the structure suffering from accidental damages or unconventional damages can be recovered to the state with ability of completing the specified missions by means of effective repairs according to the specified procedures and methods within the specified time and under the specified conditions.

For the sake of simplicity, structural durability degree, availability degree, safety degree, livability degree, survivability degree and recoverability degree can be thought as independent parameters. If the influence of each parameter on structural dynamic operational integrity is simply expressed in a linear relationship, the model of structural operational integrity degree can be expressed as follows:

$$
I_{s o}=U_{s} \cdot A_{s} \cdot S_{s} \cdot C_{s} \cdot S_{s u} \cdot R_{s c}
$$

According to the above models, the "cask effect" for structural dynamic operational integrity is very clear. As long as one of the parameters above is very small, the structural dynamic operational integrity will be greatly affected. Therefore, under the certain limited resources (such as funds, design level, supportability, etc.), the durability degree, availability degree, safety degree, livability degree, survivability degree and recoverability degree of the structure can be coordinated with each other through reasonable resources control, so as to achieve the highest structural dynamic operational integrity.

For the characterization of aircraft structural durability degree $U_{s}$, availability degree $A_{s}$, safety degree $S_{s}$, livability degree $C_{s}$ survivability degree $S_{s u}$ and recoverability degree $R_{s c}$, some expressions were shown in [23-24]. In order to discuss the characterization of structural operational integrity of common equipment structures systematically, some parameters are shown here similarly and briefly.

\subsection{Characterization of structural durability—structural durability degree, $U_{S}$}

Structural durability reflects the durable level of a kind of structures. It refers to the structural ability to 
resist failures caused by service environments, regular loads or accidental damages. Structural durability doesn't only reflect the structural reliability, but also reflects the economic efficiency of structures.

Damage degree $D_{s}$ of a kind of structures can be used to measure the severity of the damage in durable life of the kind of structures. It is a quantitative measurement of the damage generated when this kind of structures reach the specified durable time $t$, and the calculation method is as follows:

$$
D_{S}=\frac{\bar{L}_{S}(t)}{N_{S}}
$$

where, $\bar{L}_{s}(t)$ refers to the number of structures that cannot be kept sound (or operated normally) and whose functions are weakened when the specified durable time $t$ is reached, and $N_{s}$ refers to the total number of the certain kind of structures. Therefore, structural durability degree $U_{s}$ can be used to measure the durability of this kind of structures:

$$
U_{s}=1-\frac{\bar{L}_{s}(t)}{N_{s}}
$$

Also, in practice, the durable life of a kind of structures is often used to quantitatively measure structural durability, which is a limit of service period of a kind of structures with high level of reliability degree and confidence coefficient ${ }^{[25]}$.

\subsection{Characterization of structural supportability—structural availability degree, $A_{s}$}

Aircraft availability degree is often used to measure aircraft supportability ${ }^{[26]}$. Similarly, the structural availability degree $A_{s}$ is used to measure structural supportability, and only the inherent structural availability degree is used to characterize structural supportability for a brief analysis.

Inherent structural availability degree, $A_{s i}$, is the ratio of the actual work time to the total service time of structure. If the probability density function of fault is $b(t)$, and the maintenance time density function is $m(t)$, the fault interval time, $T_{u}$, and maintenance time, $T_{m}$, can be expressed respectively:

$$
T_{u}=\int_{0}^{\infty} t b(t) d t
$$

$$
T_{m}=\int_{0}^{\infty} t m(t) d t
$$

then inherent structural availability degree is,

$$
A_{s_{i}}=\frac{T_{u}}{T_{u}+T_{m}}
$$

Therefore, structural availability degree is

$$
A_{s}=A_{\text {si }}
$$

\subsection{Characterization of structural safety-structural safety degree, $S_{S}$}

Aircraft safety degree is often used to measure aircraft safety ${ }^{[27]}$. Similarly, structural safety degree, $S_{s}$, is used to measure structural safety. When the structure performs and completes the specified tasks under the specified conditions in the specified time cycle, the probability, $P$, of accidents of structure can be expressed as follows,

$$
P=R \cdot 0+(1-R) \cdot P_{A / F}
$$

where, $R$ is the structural reliability; $P_{A / F}$ is the probability of accidents when the structure fails. Therefore, structural safety degree, $S_{S}$, can be expressed as follows,

$$
S_{S}=1-P=1-(1-R) \cdot P_{A / F}
$$


If $P_{\bar{A} / F}=1-P_{A / F}$ is defined as the probability without accidents of structure, when the structure fails, the above formula can be converted into

$$
S_{S}=R+(1-R) \cdot P_{\bar{A} / F}
$$

\subsection{Characterization of structural performance — structural livability degree, $C_{S}$}

It is well known that structural system consists of structural members and forms equipment body, bearing and transferring forces in structural operational processes. So, the basic functions of structure are to bear and transfer forces in structural operational processes. Because of the limited space, material and weight requirements, structures have to carry on other functions. For example, integral tanks as well as movement mechanisms are often constructed as parts of aircraft structures. Generally, structural performance includes strength, rigidity, stability, sealability and movement discussed above.

Structural livability degree can be employed to measure structural performance. Any structure has the performance needed to achieve based on design and manufacture. That is to say that structures should be able to bear the designed loads. However, in the actual service and operational processes, for the structure which has been produced and delivered for service, its ability to bear loads is a random variable, so is the actual loads carried by the structure in service. It is inevitable that even in the regular service processes, there will be structural failures and damages as the ability to bear loads is less than the actual acting loads. Therefore, structural load-bearing ability acting load interference model can be employed to describe structural livability degree under normal operations. The schematic relationship is shown in Figure 6.

If the one-dimensional probability density function of the acting load amplitude $l_{a}$ and load-bearing ability amplitude $L_{a}$ of the structure are $f\left(l_{a}\right)$ and $g\left(L_{a}\right)$ respectively, the probability that $L_{a}$ is less than $l_{a}$ corresponding to any acting load amplitude $l_{a}$ is,

$$
G\left(l_{a}\right)=P\left(L_{a}<l_{a}\right)=\int_{0}^{l_{a}} g\left(L_{a}\right) d L_{a}
$$

Since the acting load amplitude is always non negative, the lower limit of integration is zero. The distribution function $G\left(l_{a}\right)$ is shown as the shaded area in Figure 6. The probability of acting load amplitude $l_{a}$ as a random variable is $f\left(l_{a}\right) d l_{a}$. Since $G\left(l_{a}\right)$ is a monotone function of $l_{a}$ and also a random variable, the probability of $G\left(l_{a}\right)$ occurrence is also $f\left(l_{a}\right) d l_{a}$. The product of the two is the differential, $d F_{s}$, of structural failure probability, $F_{s}$,

$$
d F_{s}=G\left(l_{a}\right) f\left(l_{a}\right) d l_{a}
$$

By integrating the above formula, structural failure probability, $F_{s}$, is obtained,

$$
F_{s}=\int_{0}^{\left(l_{a}\right)_{\max } \int} G\left(l_{a}\right) f\left(l_{a}\right) d l_{a}
$$

Then, taking equation (14) into the above equation to get,

$$
F_{s}=\int_{0}^{\left(l_{a}\right)_{\max } \int}\left[\int_{0}^{l_{a}} g\left(L_{a}\right) d L_{a}\right] f\left(l_{a}\right) d l_{a}
$$




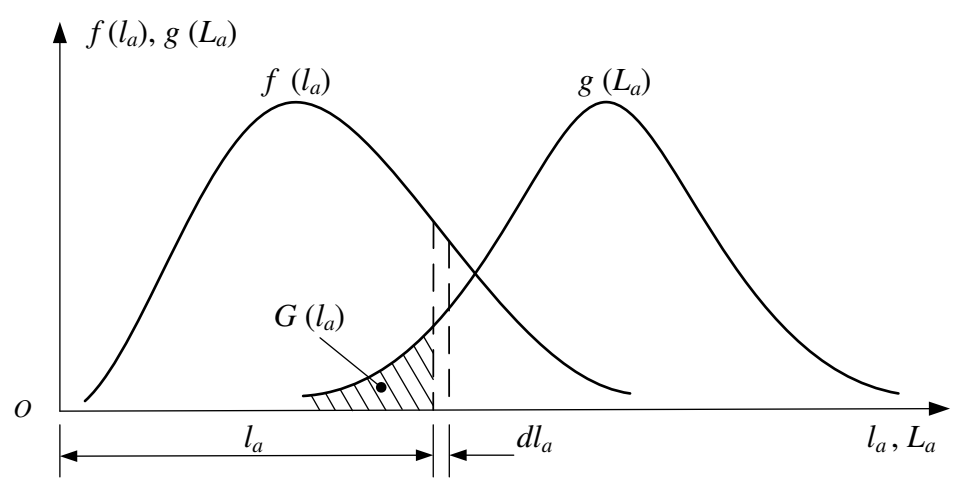

Figure 6. Load-bearing ability - acting load interference model of structure.

Therefore, structural livability degree is obtained,

$$
C_{s i}=1-F_{S}
$$

It should be noted that the last structural livability degree model is a general model. For structural strength, rigidity, stability, sealability and movement performances, load-bearing ability acting load interference models of structure will be different, and structural strength livability degree $C_{S S}$, structural rigidity livability degree $C_{r s}$, structural stability livability degree $C_{b s}$, structural sealability livability degree $C_{l s}$, and structural movement livability degree $C_{m s}$ can be obtained separately. Finally, structural livability degree $C_{S}$ in structural service or operational processes can be determined as the minimum value of these parameters, shown as follows,

$$
C_{s}=\min \left\{C_{s s}, C_{r s}, C_{b s}, C_{l s}, C_{m s}\right\}
$$

\subsection{Characterization of structural survivability — structural survivability degree, $S_{s u}$}

Susceptibility and vulnerability are two main elements that determine the survivability of aircraft ${ }^{[18]}$. Similarly, structural susceptibility and vulnerability can be used as two main elements that determine the structural survivability. Structural susceptibility is generally expressed by means of the hit probability, $P_{H}$, by threatened mechanisms such as weapon attacks, accidental crashes, hailstone-strikes, lighting strikes, birds strikes and so on. Vulnerability is a conditional probability, $P_{K / H}$, which is the damage probability when a structure is hit by one or more mechanisms. It is also equal to the ratio of the number of killed structures to the number of hit structures,

$$
P_{K / H}=M_{\text {Killed }} / M_{\text {Hit }}
$$

In above formula, $M_{\text {Killed }}$ is the number of lost structures while $M_{H i t}$ is the number of hit structures. Therefore, after the structure takes part in service, its structural damage probability is,

$$
P_{S D}=P_{H} \cdot P_{K / H}
$$

Thus, structural survivability degree is,

$$
S_{s u}=1-P_{S D}
$$

\subsection{Characterization of structural recoverability—structural recoverability degree, $R_{s c}$}

Structural recoverability can be measured by structural recoverability degree, which is a probability index and mainly reflected in recovery time, recorded as $R_{S c}(t)$, simplified as $R_{S c}$ here ${ }^{[19]}$. The meaning of structural recoverability degree, $R_{S c}$, is the probability that the structure suffering from accidental and 
unconventional damages can be restored to the state with ability of performing and completing the specified tasks in accordance with the specified procedures and methods within the specified time under the specified conditions. Also, it can be expressed with the proportion of the number of structures recovered to the state with ability of performing and completing the specified tasks. That is, structural recoverability degree, $R_{S c}$, can be expressed by the following formula,

$$
R_{s c}=P(T \leq t)=\frac{q_{t}-q_{n, t}}{q_{t}}
$$

where $T$ is the recovery time under the specified conditions, procedures and methods; $t$ is the specified time; $q_{t}$ and $q_{n, t}$ refer to the total number of structures suffering from accidental or unconventional damages within the specified time $t$ and the number of structures which are unable to be recovered to the state with specified abilities.

Also, structural recoverability can be expressed by the mean time to recovery, MTTRC, which is the average time taken by a required group of persons with the required technical levels to repair the same type structures with a certain level of accidental and unconventional damages to restore specified abilities or functions based on the specified procedures and resources (equipment, tools, spare parts, manpower, logistics, etc.). And structural recoverability can be characterized by accidental damage recovery cost $M R C C$ (Mean Recovery Cost). MRCC is actually the mean amount of resources expended by personnel with specified technical levels to repair the same type structures with a certain extent of accidental or unconventional damages to the status with specified abilities or functions by means of the prescribed procedures and methods. Meanwhile, structural recoverability can be characterized by the mean work time to recovery, MWTTRC (Mean Work Time to Recovery), which is the average working time taken by a required group of persons with the required technical levels to repair the same type structures with a certain level of accidental or unconventional damages to restore specified abilities or functions based on specified procedures and resources. Usually, the factors $M R C C$ and $M W T T R C$ can be employed to compare the recoverability of similar models of structures. And these three parameters were discussed in [28] in details.

It should be pointed out that PHM technologies can also improve structural operational integrity degree, $I_{s o}$, by means of improving structural durability degree, $U_{s}$, availability degree, $A_{s}$, safety degree, $S_{S}$, livability degree, $C_{s}$, and recoverability degree, $R_{s c}$, of structures. That is to say that PHM technologies can improve structural dynamic operational integrity, and this will be discussed in other work in details.

From the discussion above, it can be seen that structural readiness rate, $R_{s}$, and structural health degree $H_{S}(t)$ represent structural static operational integrity, also the overall and intuitive results of structural operational integrity, but it cannot reflect the direct relationship between it and other general quality characteristic parameters of structures, and it cannot tell how to improve it by means of effective design methods either. Structural operational integrity degree, $I_{s o}$, can be used to represent structural dynamic operational integrity, and it can reflect the relationship between it and other general quality characteristic parameters of structures, showing that structural dynamic operational integrity conforms to "cask effect", so as to realize the optimum design and control of structural operational integrity.

\section{Structural optimum design based on structural operational integrity}


From the discussion above, structural operational integrity degree, $I_{s o}$, can be comprehensively characterized and functioned by means of structural durability degree, $U_{s}$; availability degree, $A_{s}$; safety degree, $S_{s}$; livability degree, $C_{s}$; survivability degree, $S_{s u}$, and recoverability degree, $R_{s c}$. It can be seen that, in structural operational integrity, the deficiency of index factors above in any aspect will lead to the overall low level of structural operational integrity. That is to say, structural operational integrity index system conforms to the "cask effect", which must be considered comprehensively and coordinately in the whole life cycle and operational processes of structure. In structure design, it is not good to pursue one general quality characteristic parameter of structure. It is necessary to make structural operational integrity degree, $I_{s o}$, reach the maximum value by means of optimum design methods. This should be the final goal!

For example, in the past, in order to get higher reliability and maintainability of aircraft structure, monolithic structural components were widely employed. On the other hand, in order to obtain the higher recoverability of aircraft structure, modular and combinatorial construction should be adopted for aircraft structure. This is very contradictory! A design example of an aircraft backswept wing structure constructed by ribs, stringers and skins is shown in Figure 7.

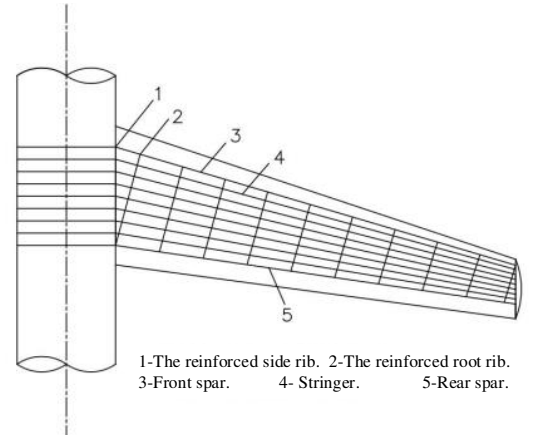

(a) Typical monolithic backswept wing structure.

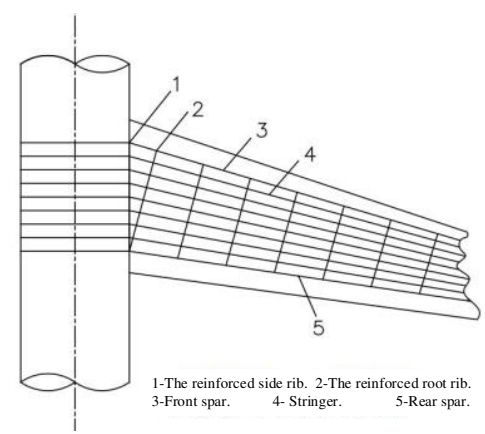

(b) Monolithic backswept wing structure with damaged wing tip.

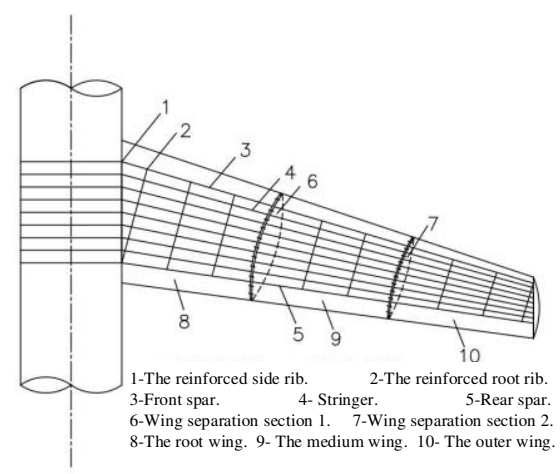

(c) Operational integrity optimally designed backswept wing structure.

Figure 7. A simple optimum design example of an aircraft backswept wing structure.

The aircraft wing shown in Figure 7 (a) is typical of a monolithic rear-sweeping wing structure without optimum structural operational integrity design. The wing consists of typical wing ribs, spars stringers and skins. Under normal service conditions, the force and transmission characteristics of the wing are good. However, when an accidental damage occurs in one part of the wing, i.e., a collision that breaks the wing tip with a large area of skin cracking and stringers broken, resulting in more serious damages, as shown in Figure 7 (b). The damaged wing needs to be recovered in accordance with the following steps: firstly, the damaged stringers and skin structures are cut away, then the damaged stringers are removed and replaced by 
new compartments with reliable connections, finally the new skins are covered. This process shows that the repair process is complex; the different types of repair tools will be required; the skill level of the repair personnel should be high as well. All of these will cause long recovery time and high repair cost. That means the aircraft structural recoverability degree, $R_{S c}$, is low, resulting in low aircraft structural operational integrity degree, $I_{s o}$. After the optimum structural design based on structural operational integrity, the aircraft wing can be designed as shown in Figure 7 (c). This aircraft wing consists of few connected parts. When the wing suffers the same collision damages as before, the best repair method is to replace the outer wing part, and this should be much easier. Of course, the actual situation is much more complicated and serious than these descriptions in practice, because some index elements work continuously at several stages of aircraft operational processes. After all, it still has a lot of work to do for the optimum structural design based on structural operational integrity.

\section{Summary}

(1) After discussing equipment integrity which categorized into equipment manufacture integrity, equipment storage integrity and equipment operational integrity, from the perspective of performing and completing the functions and missions of structures, the putting forward process and basic concept of structural operational integrity(SOI) is expounded, which is the development of the traditional aircraft structural integrity(ASI) and can be used to describe the comprehensive quality characteristics of structures in whole service period or operational processes.

(2) Structural operational integrity(SOI) can be categorized into structural static operational integrity(SSOI) and structural dynamic operational integrity (SDOI), expressing the status which exists when a structure is sound and unimpaired in service or operational process and the ability which exists when a structure is sound and unimpaired while providing the desired levels of structural durability, supportability, safety, performance, survivability and recoverability during service period or in operational processes separately.

(3) The characterizations of structural static operational integrity (SSOI) and structural dynamic operational integrity (SDOI) are presented, while the characteristics of structural operational integrity are analyzed, as well as the measurement methods of durability, supportability, safety, performance, survivability and recoverability of structures discussed.

(4) It stated that PHM technologies can improve structural operational integrity, and the optimum design method of structural operational integrity is described as well as an example of a simple optimum structural design of aircraft wing shown here.

With the employment of structural operational integrity (SOI), the structural design idea will be changed. It can help us to get lighter, low life cycle cost, durable, fast recovery and more reliable structures. The discussion results of structural operational integrity in this paper can be applied to other engineering structures such as building structures, bridge structures, infrastructure bodies, etc. Of course, it has a lot of work to do in this field. 


\section{References}

[1] Maozhi Gan, Zhenzhen Wu. Maintainability Design and Verification[M]. Beijing: National Defense In-dustry Press, 1995.

[2] Yuting He, Teng Zhang Binlin Ma, et.al. Structural integrity control technology based on structural dam-age monitoring[C]. Poland:36th Conference and 30th Symposium of the International Committee on Aer-onautical Fatigue and Structural Integrity. 2019.06.

[3] Yuting He, Teng Zhang Binlin Ma. Basic Connotation and Evaluation of Military Aircraft Structural Oper-ational Integrity [J]. Journal of Air Force Engineering University(Natural Science Edition),2019, 20(4): 433-439.

[4] USA Department of Defense. Aircraft structural integrity program(ASIP)[S]. Department of defense standard MIL-STD1530D(USFA), 2016.

[5] Baoping Qiang. Aircraft structure strength ground test[M]. Beijing: Aviation industry press, 2014: 29-102.

[6] The Welding Institute (TWI). What Is Structural Integrity and Why Is It Important?[EB/OL]. 2021: https://www.twiglobal.com/technical-knowledge/faqs/structural-integrity.

[7] He Yuting. A study of equipment operational integrity. Journal of Air Force Engineering University (Nat-ural Science Edition), Vol. 21, No. 1, pp 1-8,2020.

[8] Yuting He. On Aircraft Operational Integrity [C]. China: 32nd Congress of the International Council of the Aeronautical Sciences. 2021.9.

[9] Wenting Liu, Yuhai Li, Qunzhi Chen, et al. Accelerated Corrosion Environmental Spectrums for Testing Surface Coatings of Critical Areas of Flight Aircraft Structures [J]. Journal of Beijing University of Aero-nautics and Astronautics, 2002 (01): 109-112.

[10] http://k.sina.com.cn/article_6445346154_1802c2d6a00100fw2s.html?cre=tianyi\&mod=pcpager_focus\&loc=23\&r=.

[11] Youjun Huang. Damage treatment of extensive fatigue cracks on the rear lower fuselage skin of Boeing 737CL aircraft[J].Aviation Maintenance and Engineering, 2015, 000(007):52-55.

[12] Yuhai $\mathrm{Li}$, Feng Shi. Failure analysis of fretting damage to the torsion tube of the flap drive system of Boeing 747[C].//Chinese Society of Aeronautics and Astronautics Symposium on Mechanical Fretting Damage, 1994.

[13] https://baijiahao.baidu.com/s?id=1667398522029748817\&wfr=spider\&for=pc.

[14] The General Armaments Department of PLA. Quality Management System Requirements[S]. The People's Republic of China military standard, GJB 9001C-2017, 2017.

[15] Haijun Zhang. Review on Quality Characteristic of Equipment[J]. Dual Use Technologies \& Products, 2015, 09(2): $252-$ 253.

[16] The General Armaments Department of PLA. Terminology of Reliability and Maintainability[S]. The Peo-ple's Republic of China military standard, GJB451.A-2005, 2005.

[17] Shulin LI, Fei Chang, Yuting He, et.al. Survivability Analysis and Evaluation of Military Aircraft in Op-erational processes[M]. Beijing: National Defense Industry Press, 2016.

[18] The General Armaments Department of PLA. Structural integrity program of military aircraft. The Peo-ple's Republic of China military standard, GJB775.A-2012, 2012.

[19] Yuting He. Aircraft Recoverability and Its Design Methods[J]. Journal of Air Force Engineering Universi-ty(Natural Science Edition),2020, 21(1).

[20] Jianhua Zhang. Aircraft Battle Injury Emergency Repair Engineering[M]. Beijing: Aviation Industry Press, 2001.

[21] Wuwen Yao. Criteria and Methods for Safety Flight Assessment of Battle Injured Aircraft[M]. Beijing: Na-tional Defense Industry Press, 2016.

[22] Yuting He. Study on characterization method of aircraft health status[J]. Advances in Aeronautical Sci-ence and Engineering, 2021,12(3):1-8.(in Chinese).

[23] Hengxi Zhang, Xiaodong Liu, Baojun Duan, et al. Cost-effectiveness Analysis of Modern Aircraft[M]. Bei-jing: Aviation Industry Press, 2001.

[24] Shaoming Ma, Guodong Zhang. Comprehensive Support Project[M]. Beijing: National Defense Industry Press, 1995.

[25] Yuting He, et.al. Theory and Technology of Aircraft Structural Life Control[M]. Beijing: National Defense Industry Press, 2017. 
[26] Yuting He. On The Equipment Operational Integrity[J]. Journal of Air Force Engineering Universi-ty(Natural Science Edition),2020, 21(2):1-8.

[27] Yuting He. Safety Engineering of Aircraft[M]. Beijing: National Defense Industry Press, 2014.

[28] Yuting He. On the Evaluation and Design Methods of Structural Recoverability[C]. Korea: 2021 Asia-Pacific International Symposium on Aerospace Technology. 2021.11.

\section{Availability of data and materials}

Not applicable.

\section{Competing interests}

The author declares that they have no competing interests.

\section{Funding}

The author is grateful for the supports of National Science and Technology Major Special Funding (No: J2019-I-0016-0015) and the National Natural Science Foundation of China (52175155).

\section{Authors' contributions}

Yuting He was the sole contributor to the writing of the manuscript.

\section{Acknowledgments}

Not applicable. 